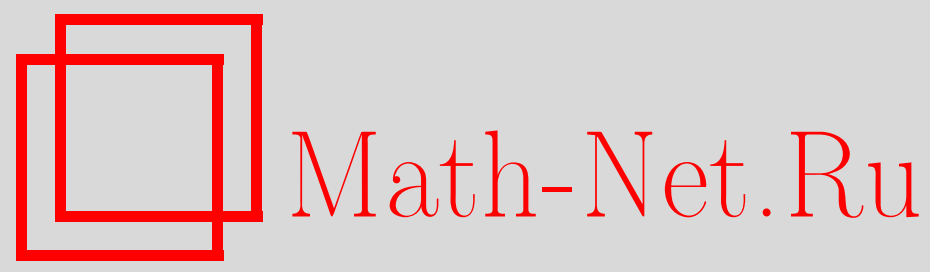

Ю. В. Нестеренко, Об одном тождестве Гильберта, Матем. заметки, 1999, том 66, выпуск 4, 527-532

DOI: https://doi.org/10.4213/mzm1196

Использование Общероссийского математического портала Math-Net.Ru подразумевает, что вы прочитали и согласны с пользовательским соглашением http://www .mathnet.ru/rus/agreement

Параметры загрузки:

IP : 54.164 .48 .24

26 апреля 2023 г., 09:38:31

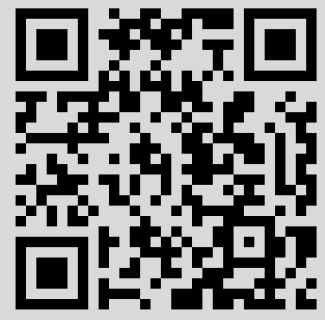




\title{
ОБ ОДНОМ ТОЖДЕСТВЕ ГИЛЬБЕРТА
}

Ю.В. Нестеренко

\begin{abstract}
В статье приводится простое доказательство полиномиального тождества, использовавшегося Д. Гильбертом при решении проблемы Варинга. Доказательство основано на разложении в непрерьвную дробь одного формального гипергеометрического ряда.
\end{abstract}

Библиографиял: 11 названий.

В 1770 году Ж. Л. Лагранж доказал, что каждое натуральное число есть сумма не более четырех квадратов натуральных чисел. В том же году Е. Варинг высказал предположение, что каждое натуральное число есть сумма не более девяти кубов натуральных чисел, не более девятнадиати биквадратов и т.д.

Первьй шаг в решении этой проблемы сделал в 1859 году Ж. Лиувилль, доказавший, что каждое натуральное число есть сумма не более 53 биквадратов. При этом он использовал некоторое тождество для многочленов от четырех переменных. Позднее для этой же цели Е. Люка предложил более простое тождество

$$
6\left(x_{1}^{2}+x_{2}^{2}+x_{3}^{3}+x_{4}^{2}\right)^{2}=\sum_{i, j}\left(\left(x_{i}+x_{j}\right)^{4}+\left(x_{i}-x_{j}\right)^{4}\right),
$$

в котором суммирование ведется по всем парам целых чисел $i, j$ с условием $1 \leqslant i<j \leqslant 4$. Впоследствии усилиями многих математиков подобные результаты были доказаны для $n=5,6,7,8,10$ (см. [1]). При этом в основе доказательств лежали все более сложные тождества, подобные (1). Так, например, А. Гурвитц, доказавший, что каждое натуральное число есть сумма не более чем 36119 восьмых степеней натуральных чисел [2], использовал тождество, правая часть которого содержит 184 слагаемых.

В 1909 году Д. Гильберт доказал [3], что каждое натуральное число допускает представление в виде суммы $n$-x степеней натуральных чисел, количество которых не превосходит некоторой границы, определяемой только показателем $n u$ не зависящей от представляемого числа.

Принято считать, что этим проблема Варинга была решена. Распространено мнение, что доказательство Гильберта весьма сложно в техническом отношении и трудно для понимания. В действительности это не так. Работа Гильберта завершает цельй ряд исследований, естественньм образом обобщая и развивая предшествующие идеи. Основные технические трудности сосредоточены в доказательстве формулируемой ниже теоремы 1. Вывод из нее гипотезы Варинга совершенно элементарен.

Работа выполнена при частичной поддержке Российского фонда фундаментальных исследований, грант № 97-01-00181. 
ТЕорема 1. Для любых иелых $m>1 u r>1$ существуют положительные рациональные числа $b_{i}$ и иелые числа $a_{i j}$ такие, что тождественно по $r$ переменным $x_{1}, \ldots, x_{r}$ выполняется равенство

$$
\left(x_{1}^{2}+\cdots+x_{r}^{2}\right)^{m}=\sum_{j=1}^{M} b_{j}\left(a_{1 j} x_{1}+\cdots+a_{r j} x_{r}\right)^{2 m},
$$

причем $M=(2 m+1)^{r}$.

Отметим, что Гильберт доказал эту теорему при $r=5$ и меньшем значении $M$. Однако для решения проблемы Варинга в его формулировке величина $M$ не имеет значения.

Теорема 1 была сформулирована в работе Гурвитца [2] в качестве предположения и использовалась там для доказательства утверждения о том, что из справедливости гипотезы Варинга для показателя $m$ следует ее справедливость для показателя $2 m$. Впервые теорема 1 была доказана Гильбертом [3] с использованием для этого кратных интегралов по многомерному шару. Одновременно со статьей Гильберта была опубликована статья Хаусдорфа [4], где давалось иное доказательство теоремы, применяшее кратные несобственные интегралы и ортогональные многочлены Эрмита. Впоследствии оказалось, что доказательство Хаусдорфа может быть проведено без использования интегралов. За три года в работах Е. Стридсберга [5], А. Гурвитца [6], Р. Ремака [7], Г. Фробениуса [8] был найден элементарньй вариант рассуждений Хаусдорфа. В предлагаемой статье дается еще одно элементарное доказательство теоремы 1 . По существу оно также является переработкой доказательства Хаусдорфа.

Определим последовательность целых чисел $c_{k}$ равенствами

$$
c_{2 j}=\frac{(2 j) !}{j !}, \quad c_{2 j+1}=0, \quad j \geqslant 0 .
$$

Пусть при некоторых натуральных $n$ и $m$ действительные числа $\alpha_{h}, \beta_{h}, h=1, \ldots, n$, удовлетворяют условиям

$$
\sum_{h=1}^{n} \beta_{h} \alpha_{h}^{k}=c_{k}, \quad k=0,1, \ldots, 2 m, \quad \beta_{h}>0 .
$$

Тогда тождественно по переменным $x_{1}, \ldots, x_{r}$ выполняются равенства

$$
\begin{aligned}
& \sum_{h_{1}=1}^{n} \cdots \sum_{h_{r}=1}^{n} \beta_{h_{1}} \cdots \beta_{h_{r}}\left(\alpha_{h_{1}} x_{1}+\cdots+\alpha_{h_{r}} x_{r}\right)^{2 m} \\
& \quad=\sum_{h_{1}=1}^{n} \cdots \sum_{h_{r}=1}^{n} \sum_{k_{1}+\cdots+k_{r}=2 m} \frac{(2 m) !}{k_{1} ! \cdots k_{r} !} \beta_{h_{1}} \cdots \beta_{h_{r}} \alpha_{h_{1}}^{k_{1}} \cdots \alpha_{h_{r}}^{k_{r}} x_{1}^{k_{1}} \cdots x_{r}^{k_{r}} \\
& \quad=\sum_{k_{1}+\cdots+k_{r}=2 m} \frac{(2 m) !}{k_{1} ! \cdots k_{r} !} c_{k_{1}} \cdots c_{k_{r}} x_{1}^{k_{1}} \cdots x_{r}^{k_{r}} \\
& \quad=\frac{(2 m) !}{m !} \sum_{j_{1}+\cdots+j_{r}=m} \frac{(m) !}{j_{1} ! \cdots j_{r} !} x_{1}^{2 j_{1}} \cdots x_{r}^{2 j_{r}}=\frac{(2 m) !}{m !}\left(x_{1}^{2}+\cdots+x_{r}^{2}\right)^{m}
\end{aligned}
$$

Таким образом, теорема 1 будет доказана, если мы установим, что при некотором $n$ система условий (3) относительно неизвестных $\alpha_{h}, \beta_{h}$ разрешима в рациональных числах. 
Положим в $(3),(4)$ параметр $n=2 m+1$. Тогда для доказательства теоремы достаточно установить сушествование действительных чисел $\beta_{k}$ и различных действительных чисел $\alpha_{k}$, удовлетворяющих условиям (3). Действительно, при фиксированных различных $\alpha_{h}$ и $n=2 m+1$ равенства (3) определяют числа $\beta_{k}$ единственным образом. При этом числа $\beta_{k}$ непрерывно зависят от $\alpha_{h}$. Если выбрать рациональные числа $\alpha_{h}^{\prime}$ достаточно близкими к $\alpha_{h}$ и определить для них соответствующие числа $\beta_{k}^{\prime}$ с помощью равенств (3), то числа $\beta_{k}^{\prime}$ также будут расположены вблизи от чисел $\beta_{k}$. Это означает, что для рациональных чисел $\alpha_{k}^{\prime}, \beta_{k}^{\prime}$ будут вьполнены все условия (3) и, следовательно, теорема 1 будет справедлива.

Определим формальные ряды $f_{n}(x)$ следуюшим образом:

$$
f_{n}(x)=2^{n} \sum_{k=0}^{\infty} \frac{(2 k+n) !}{k !} x^{-2 k-n-1}, \quad n \geqslant 0 .
$$

Тогда

$$
f_{0}(x)=\sum_{k=0}^{\infty} c_{k} x^{-k-1} .
$$

Лемма 1. Справедливы следующие соотношения:

$$
\begin{aligned}
f_{1}(x) & =x f_{0}(x)-1, \\
f_{n+1}(x) & =x f_{n}(x)-2 n f_{n-1}(x), \quad n \geqslant 1 .
\end{aligned}
$$

ДокАЗАТЕЛЬСТво. Оба тождества (6), (7) доказываются сравнением коэффициентов соответствующих рядов. Имеем

$$
x f_{0}(x)-1=2 \sum_{k=1}^{\infty} \frac{(2 k-1) !}{(k-1) !} \cdot x^{-2 k}=f_{1}(x)
$$

и при $n \geqslant 1$

$$
x f_{n}(x)-2 n f_{n-1}(x)=x^{-n} 2^{n+1} \sum_{k=1}^{\infty} \frac{(2 k+n-1) !}{(k-1) !} \cdot x^{-2 k}=f_{n+1}(x) .
$$

Определим последовательность многочленов $Q_{n}(x)$ с помощью рекуррентного уравнения

$$
Q_{n+1}(x)=x Q_{n}(x)-2 n Q_{n-1}(x), \quad Q_{0}(x)=1, \quad Q_{1}(x)=x .
$$

Вторую последовательность многочленов $P_{n}(x)$ определим с помощью того же рекуррентного уравнения, но с начальными данными $P_{0}(x)=0, P_{1}(x)=1$. Из (8) следует, что степень многочлена $Q_{n}(x)$ равна $n$, а коэффициент при $x^{n}$ равен 1 . Кроме того, $\operatorname{deg} P_{n}(x) \leqslant n-1$.

ЛЕмма 2. При $n \geqslant 0$ справедливы тожсдества

$$
\begin{gathered}
f_{n}(x)=Q_{n}(x) f_{0}(x)-P_{n}(x), \\
P_{n+1}(x) Q_{n}(x)-P_{n}(x) Q_{n+1}(x)=2^{n} \cdot n !, \\
Q_{n+1}^{\prime}(x)=(n+1) Q_{n}(x) .
\end{gathered}
$$


ДокАЗАТЕЛЬСТво. Три последовательности $f_{n}(x), P_{n}(x), Q_{n}(x)$ удовлетворяют одному и тому же рекуррентному уравнению второго порядка (см. (7), (8)). Поэтому для доказательства (9) достаточно проверить эти соотношения при $n=0,1$. При $n=0$ равенство (9) следует из определения многочленов $P_{0}(x)$ и $Q_{0}(x)$, а при $n=1$ оно совпадает с равенством (6).

Прямое вычисление показывает, что $P_{1}(x) Q_{0}(x)-P_{0}(x) Q_{1}(x)=1$. Так как обе последовательности $P_{n}(x)$ и $Q_{n}(x)$ удовлетворяют рекуррентному уравнению (8), справедливо тождество

$$
P_{n+1}(x) Q_{n}(x)-P_{n}(x) Q_{n+1}(x)=2 n\left(P_{n}(x) Q_{n-1}(x)-P_{n-1}(x) Q_{n}(x)\right),
$$

из которого с помощью индукции получается равенство (10).

Для доказательства (11) также воспользуемся индукцией. При $n=0$ и $n=1$ равенство (11) проверяется непосредственно. Предположим, что оно справедливо при всех $n<k$, где $k \geqslant 2$. Дифференцируя равенство

$$
Q_{k+1}(x)=x Q_{k}(x)-2 k Q_{k-1}(x)
$$

и пользуясь предположением, находим

$$
Q_{k+1}^{\prime}(x)=Q_{k}(x)+x k Q_{k-1}(x)-2 k(k-1) Q_{k-2}(x)=(k+1) Q_{k}(x) .
$$

Лемма 3. Для $n \geqslant 1$ многочлен $Q_{n}(x)$ имеет $n$ действительных корней $\lambda_{1}<$ $\lambda_{2}<\cdots<\lambda_{n}$, причем

$$
\operatorname{sign} Q_{n}^{\prime}\left(\lambda_{j}\right)=(-1)^{n-j}, \quad j=1, \ldots, n .
$$

ДокАЗАТЕЛЬСтво. Докажем лемму индукцией по $n$. При $n=1$ имеем $Q_{1}(x)=x$ и утверждение, очевидно, выполняется. Докажем теперь утверждение леммы для многочлена $Q_{n+1}(x)$, считая, что для $Q_{n}(x)$ оно имеет место. Из равенств $(8)$ и $(11)$ находим

$$
Q_{n+1}\left(\lambda_{j}\right)=-2 n Q_{n-1}\left(\lambda_{j}\right)=-2 Q_{n}^{\prime}\left(\lambda_{j}\right) .
$$

Поэтому sign $Q_{n+1}\left(\lambda_{j}\right)=(-1)^{n+1-j}$. Отсюда следует, что существуют нули $\mu_{j}$ многочлена $Q_{n+1}(x)$ такие, что

$$
\lambda_{1}<\mu_{2}<\lambda_{2}<\cdots<\lambda_{n-1}<\mu_{n}<\lambda_{n} .
$$

Учитьвая, что $\operatorname{sign} Q_{n+1}\left(\lambda_{n}\right)=-1$ и $\operatorname{sign} Q_{n+1}(+\infty)=1$, заключаем, что многочлен $Q_{n+1}(x)$ имеет нуль $\mu_{n+1}>\lambda_{n}$. Кроме того, равенства $\operatorname{sign} Q_{n+1}(-\infty)=(-1)^{n+1}$, $\operatorname{sign} Q_{n+1}\left(\lambda_{1}\right)=(-1)^{n}$ означают существование нуля $\mu_{1}$ с условием $\mu_{1}<\lambda_{1}$. Итак, многочлен $Q_{n+1}(x)$ имеет $n+1$ различных действительных нулей.

Пользуясь теперь следующими из (11) равенствами $Q_{n+1}^{\prime}\left(\mu_{j}\right)=(n+1) Q_{n}\left(\mu_{j}\right)$ и неравенствами $\mu_{1}<\lambda_{1}<\mu_{2}<\lambda_{2}<\cdots<\lambda_{n-1}<\mu_{n}<\lambda_{n}<\mu_{n+1}$, заключаем, что последовательность $Q_{n+1}^{\prime}\left(\mu_{j}\right)$ знакочередующаяся. Поскольку $Q_{n}\left(\mu_{n+1}\right)>0$ и, следовательно, $Q_{n+1}^{\prime}\left(\mu_{n+1}\right)>0$, находим, что $\operatorname{sign} Q_{n+1}^{\prime}\left(\mu_{j}\right)=(-1)^{n+1-j}$.

Завершим теперь доказательство существования действительных чисел $\alpha_{h}, \beta_{h}$, удовлетворяющих условиям (3) при $n=2 m+1$. Обозначим через $\alpha_{1}, \ldots, \alpha_{n}$ корни 
многочлена $Q_{n}(x)$. Согласно лемме 3 все они действительны и различны. Поскольку $\operatorname{deg} P_{n}(x)<\operatorname{deg} Q_{n}(x)$, рациональная функция $P_{n}(x) / Q_{n}(x)$ имеет следующее разложение в сумму простейших дробей:

$$
\frac{P_{n}(x)}{Q_{n}(x)}=\frac{\beta_{1}}{x-\alpha_{n}}+\cdots+\frac{\beta_{n}}{x-\alpha_{n}} .
$$

Из этого равенства и тождеств $(10),(11)$ находим

$$
\beta_{h}=\frac{P_{n}\left(\alpha_{h}\right)}{Q_{n}^{\prime}\left(\alpha_{h}\right)}=\frac{2^{n-1} \cdot(n-1) !}{Q_{n}^{\prime}\left(\alpha_{h}\right) Q_{n-1}\left(\alpha_{h}\right)}=\frac{2^{n-1} \cdot n !}{Q_{n}^{\prime}\left(\alpha_{h}\right)^{2}}>0 .
$$

Из (12) следует, что разложение функции $P_{n}(x) / Q_{n}(x)$ в ряд Тейлора в окрестности точки $x=\infty$ имеет вид

$$
\frac{P_{n}(x)}{Q_{n}(x)}=\sum_{k=0}^{\infty}\left(\sum_{h=1}^{n} \alpha_{h} \beta_{h}^{k}\right) x^{-k-1} .
$$

Но согласно (9) первые $2 n$ коэффициентов этого разложения совпадают с соответствующими коэффициентами ряда $(5)$ для $f_{0}(x)$. Таким образом, выполняются равенства

$$
\sum_{h=1}^{n} \alpha_{h} \beta_{h}^{k}=c_{k}, \quad k=0,1, \ldots, 2 n-1
$$

и, в частности, равенства (3) при $n=2 m+1$. Итак, действительные числа, удовлетворяющие условиям (3), построены. Это завершает доказательство теоремы 1.

ЗАмЕЧАнИЕ 1. Формальньй ряд (5), определяюший $f_{0}(x)$, является вырожденной гипергеометрической функцией, и в обозначениях [9, гл. 2] может быть записан в виде $z^{-1} \Omega\left(1 / 2,1 ;(2 z)^{-2}\right)$. Легко проверить, что он дает ассимптотическое разложение в окрестности $x=\infty$ функции

$$
\frac{1}{\sqrt{\pi}} \int_{-\infty}^{+\infty} e^{-t^{2} / 4} \frac{d t}{x-t}
$$

Разложения таких рядов в непрерывные дроби хорошо известны (см. [9, гл. 2]). Дроби $P_{n}(x) / Q_{n}(x)$ являются подходящими дробями непрерьвной дроби для $f_{0}(x)$. Рекуррентные уравнения (8) - это уравнения для числителей и знаменателей подходящих дробей (см. [9, формула (2.1.6)]). Равенства (10) - также классические соотношения между числителями и знаменателями соседних подходящих дробей (см. [9, формула (2.1.9)]). Знаменатели подходящих дробей $Q_{n}(x)$ связаны с классическими многочленами Эрмита $H_{n}(x)$ соотношением $Q_{n}(x)=H_{n}(x / 2)$ (см., например, [10, формула (63.10)]). В силу этого соотношение (11) и утверждение леммы 3 также хорошо известны.

ЗАмечАниЕ 2. Доказательство теоремы 1 из работ [5]-[8] легкоможет быть описано в терминах теории ортогональных многочленов.

Квадратичная форма

$$
\sum_{i, j=0}^{n} c_{i+j} x_{i} x_{j}
$$

положительно определена, что следует из тождества

$$
\sum_{i, j=0}^{n} c_{i+j} x_{i} x_{j}=\sum_{k=0}^{n} 2^{k} \cdot k !\left(\sum_{r=k}^{n}\left(\begin{array}{l}
r \\
k
\end{array}\right) c_{r-k} x_{r}\right)^{2}
$$


(cp. c [6]). Поэтому последовательность $c_{n}$ позитивна (см. $[11$, гл. $\left.2, \S 5]\right)$ и также позитивен линейный функционал $\mathfrak{S}$ на пространстве многочленов $\mathbb{C}[x]$, ставяший в соответствие многочлену $a(x)=a_{0}+a_{1} x+\cdots+a_{k} x^{k}$ число $\mathfrak{S}\{a(x)\}=a_{0} c_{0}+a_{1} c_{1}+\cdots+a_{k} c_{k}$ (см. $[11$, лемма 5.2]). Пользуясь явным видом многочленов Эрмита, можно непосредственньми вычислениями проверить равенства

$$
\mathfrak{S}\left\{x^{i} Q_{n}(x)\right\}=0, \quad i=0,1, \ldots, n-1,
$$

т.е. многочлены $Q_{n}(x)$ образуют ортогональное семейство относительно функционала $\mathfrak{S}$. Если $\alpha_{1}, \ldots, \alpha_{n}$ - корни многочлена $Q_{n}(x)$ и числа $\beta_{h}$ определены равенствами (3), то числа $\beta_{h}$ являются так назьваемыми коэффициентами Кристофеля и положительны (см. [11, утверждение 5.5]). Таким образом, числа $\alpha_{h}, \beta_{h}$ удовлетворяют всем условиям (3).

Отметим, что функционал $\mathfrak{S}$ может быть задан в интегральной форме

$$
\mathfrak{S}\{a(x)\}=\frac{1}{2 \sqrt{\pi}} \int_{-\infty}^{+\infty} e^{-x^{2} / 4} a(x) d x,
$$

откуда сразу следует его позитивность. Именно в таком виде, но в другой нормировке, он и использовался Хаусдорфом в [4]. Там же применялся интегральный аналог тождества (4). Само это тождество впервые появилось в работе Стридсберга [5].

\section{СПИСОК ЦИТИРОВАННОЙ ЛИТЕРАТУРЫ}

[1] Dickson L. E. History of the Theory of Numbers. V. 2. New York: Chelsea, 1971.

[2] Hurwitz A. Über die Darstellung der ganzen Zahlen als Summen von $n^{\text {ten }}$ Potenzen ganzer Zahlen // Math. Ann. 1908. V. 65. №3. P. 424-427.

[3] Hilbert D. Beweis für Darstellbarkeit der ganzen Zahlen durch eine feste Anzahl $n$-ter Potenzen (Waringsches Problem) // Math. Ann. 1909. V. 67. Р. 281-300; Гильберт Д. // Избранные труды. Т. 1. М.: Факториал, 1998. С. 312-328.

[4] Hausdorf F. Zur Hilbertschen Lözung des Waringschen Problems // Math. Ann. 1909. V. 67. P. 301-305.

[5] Stridsberg E. Sur la démonstration de D. Hilbert du théorème de Waring // Math. Ann. 1912. V. 72. № 2. P. 145-152.

[6] Hurwitz A. Über definite Polynome // Math. Ann. 1912. V. 73. P. 173-176.

[7] Remak R. Bemerkung zu Herrn Stridsbergs Beweis des Waringschen Theorems // Math. Ann. 1912. V. 72. P. 153-156.

[8] Frobenius G. Über den Stridsbergschen Beweis des Waringschen Satzes // Sitzungsber. Akad. Wiss. Berlin. 1912. P. 666-670.

[9] Джоунс У., Трон В. Непрерывные дроби. М.: Мир, 1985.

[10] Кузнецов Д. С. Специальные функции. М.: Высшая школа, 1965.

[11] Никишин Е. М., Сорокин В.Н. Рациональные аппроксимации и ортогональность. М.: Наука, 1988. 